\title{
Demonstration of Down-Chirped and Chirp-Free Pulses from High-Repetition-Rate Passively Mode-Locked Lasers
}

\author{
Randal A. Salvatore and Amnon Yariv, Fellow, IEEE
}

\begin{abstract}
Knowledge and control of the chirp parameters of semiconductor lasers is a prerequisite to obtaining transformlimited pulses and/or to compensate for group velocity dispersion in fiber. Here, we report measurements of the sign and magnitude of chirp in high-repetition-rate mode-locked semiconductor lasers. The chirp of these monolithic lasers is measured in the frequency domain, using filtering and cross-correlation techniques. For different injection currents, a range of different chirp values is measured, including strongly down-chirped pulses at higher injection currents and transform-limited pulses to slightly upchirped pulses at lower injection currents. The pulse chirp and the resulting broadening are due to the algebraic addition of opposite-signed chirps due to saturation of the absorption section and the gain section. These may cancel each other under some conditions, leading to a soliton-like transform-limited pulse.
\end{abstract}

\section{INTRODUCTION}

$\mathbf{M}$ ODE-LOCKED semiconductor lasers have produced higher repetition rate pulses than all other types of lasers. Large material gain coefficients, fast recovery times, and the ability to make short monolithic cavities allow high repetition rate pulse trains $(>50 \mathrm{GHz}$ ) to be generated easily. Repetition rates of up to $240 \mathrm{GHz}$ in GaAs-AlGaAs and above $1.5 \mathrm{THz}$ [1] in InGaAs-InGaAsP multisection structures have been reported. Although the carrier populations in both the gain and absorber sections are incapable of fully recovering during these $<20$ ps periods, a sufficient modulation in each section is obtained to couple the modes [3]. Typically in high-repetition rate mode locking, the repetition rate is larger than both the spontaneous recombination rate and average stimulated recombination rate, so the material response lags the optical pulse by nearly $\pi / 2$ radians in the repetition period. The oscillation in the gain and absorber section populations are small enough to be accurately considered as linearized modulations which produce mode coupling [3], [4]. As is known from studies in noise [5] or semiconductor laser amplifiers [6], changes in optical gain are accompanied by a material-generated phase shift due to amplitude-phase coupling characterized by the material parameter $\alpha=-\frac{d \chi_{r}}{d N} / \frac{d \chi_{i}}{d N}$.

Analysis shows that the phase modulation that results when a pulse saturates an absorbing medium has a sign opposite

\footnotetext{
Manuscript received May 10, 1995; revised June 28, 1995. This work was supported by the National Science Foundation under Grant ECS-9001272, and the Office of Naval Research under Grant N00014-91-J-1195.

The authors are with the Department of Applied Physics 128-95, California Institute of Technology, Pasadena, CA 91125 USA

IEEE Log Number 9414283.
}

to that due to saturating a gain medium [7]. In general, the passively mode-locked semiconductor laser is not likely to be purely amplitude modulated. The accompanying phase modulation typically results in a nearly linear chirp on the pulses. To our knowledge, down-chirped pulses have never been obtained from electrically-pumped passively mode-locked semiconductor lasers [8]. It would be useful for one to develop a laser which can operate in all three dispersion regimes (i.e., downchirped, chirp-free, and up-chirped) and to achieve control over its operation. In this paper, we present experimental results from a monolithic passively mode-locked laser. We find that both the magnitude and sign of chirp may be controlled. Dispersion is expected to result from self-phase modulation of both the saturable gain and absorption sections and from cavity dispersion. When using a relatively strong saturable absorber, opposite signed contributions may cancel, causing a solitonlike pulse shaping to occur [9], creating a compensated net cavity dispersion. Chirp-free operation is obtained within the monolithic cavity, producing time-bandwidth-limited pulses, without the need for any gratings or filters.

\section{EXPERIMENTAL SETUP}

A two-section, monolithic, GaAs-AlGaAs quadruple quantum-well buried heterostructure laser is used in this experiment. The laser is $510 \mu \mathrm{m}$ in length with a $390-\mu \mathrm{m}$ long gain section, a $70-\mu \mathrm{m}$ absorber section, and a $50-\mu \mathrm{m}$ isolation region between sections. The threshold was $9.5 \mathrm{~mA}$ with the gain section pumped and absorber contact floating. All mode-locked measurements presented here were done with the absorber section grounded and the gain section driven by a dc current source. Both facets were coated for a reflection of $70 \%$, and no additional feedback was added. Mode-locked measurements were obtained using light from the gain facet, however, results using the absorber facet were also measured with no noticeable difference, showing that the single-pass effects from each section were relatively small.

The phase of the optical spectrum was measured as described previously [10], [11], using a spatial filter in the Fourier plane of the dual-grating pulse compressor, shown in Fig. 1. This filtered beam was temporally cross correlated with an unfiltered, variable-delay beam from the same laser. The cross-correlation signal was obtained using a second harmonic generation (SHG) setup, and the relevant delay times versus filter center wavelengths were recorded to measure the mode- 


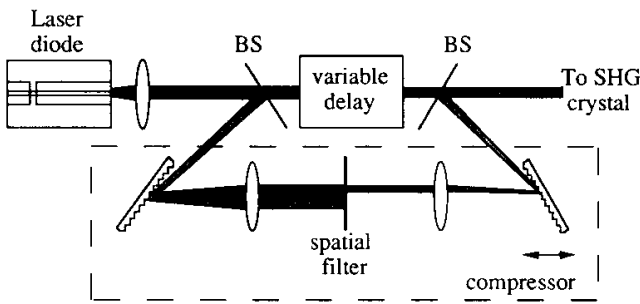

Fig. 1. Two-section monolithic passively mode-locked laser. The back contact (left in the picture) is grounded and extracts carriers from the saturable absorber section. The front contact is forward biased to inject carriers into the gain section. The laser is followed by a beam splitter. One of the beams is filtered and its pulse is cross correlated with the original pulse to measure the time delay of its peak.

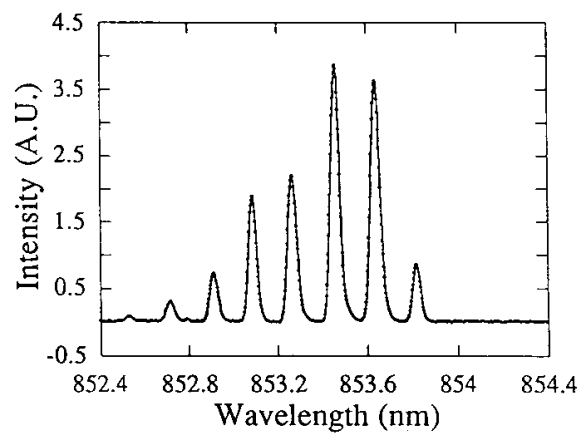

Fig. 2. Optical spectrum of mode-locked laser with absorber section grounded and $30 \mathrm{~mA}$ into gain section.

locked laser's chirp, $d \tau / d \omega$. The sliding filter width was adjusted to pass $0.5 \mathrm{~nm}$ of the pulse train's optical spectrum.

\section{EXPERIMENTAL RESULTS}

The laser was found to generate stable mode-locked pulse trains at $73 \mathrm{GHz}$, with the absorber grounded over a range of gain currents from $18 \mathrm{~mA}$ to at least $40 \mathrm{~mA}$. At the average power of $2.5 \mathrm{~mW}$ for a $30-\mathrm{mA}$ gain current, the optical spectrum had four modes within its full width at halfmaximum intensity, as shown in Fig. 2 . With the gratings placed at the focal points of the telescoped dual-grating pulse compressor, adding no additional chirp, the plot (Fig. 3) time delay of the filtered pulse versus filter center wavelength was obtained. The positive slope of the plot corresponds to a pulse in which the frequency decreases with time, i.e., a down-chirp. The magnitude of this down-chirp was found to be $1.7 \mathrm{ps} / \mathrm{nm}$ and appears essentially linear, although the spectrum is not broad enough to allow one to convincingly rule out the presence of higher order chirp.

The same chirp measurement was also made by moving the second grating to a position where the net chirp was best eliminated (i.e., the time delay versus $\lambda$ curve was made flat). This position for the second grating was found to be $5.8 \mathrm{~cm}$ inside the focal length for 2000 lines $/ \mathrm{mm}$ gratings tilted with their normal $39.7^{\circ}$ off the telescope's central axis, indicating that a down-chirp of $1.62 \mathrm{ps} / \mathrm{nm}$ was produced by the monolithic mode-locked laser. These frequency-domain chirp measurements are possible even though the measured pulses may have less than $10 \mathrm{fJ}$ per pulse. From these measurements, one would conclude that if the center-mode's

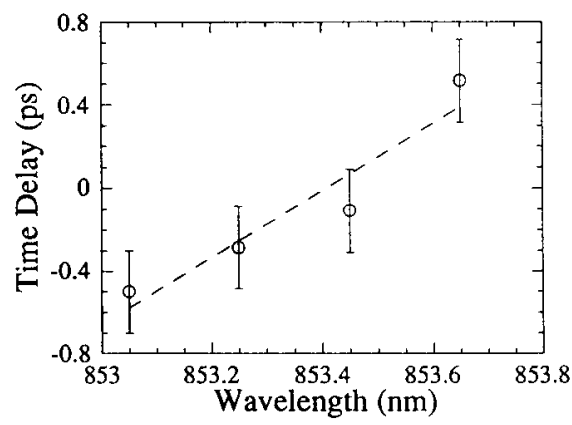

Fig. 3. Chirp measured in frequency domain, displayed in terms of the time delay of the pulse's peak versus the center wavelength of a $0.5-\mathrm{nm}$ rectangular spectral filter. The measurement is done with $30 \mathrm{~mA}$ into gain section and with absorber grounded. It shows an essentially linear down-chirp of $1.7 \mathrm{ps} / \mathrm{nm}$.

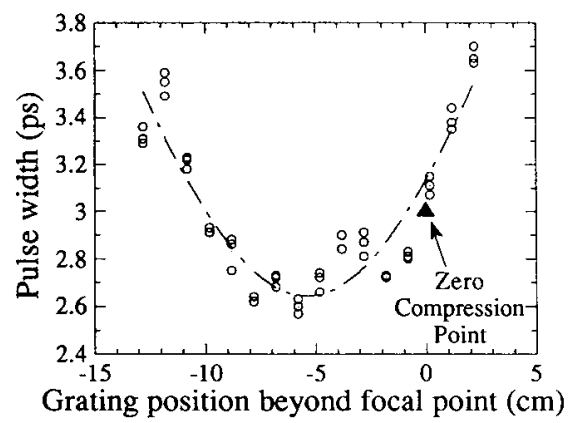

Fig. 4. Pulse width versus position of second grating in dual-grating compressor is shown. A position of zero indicates the grating is at the focal point and no chirp is added. Negative positions indicate the grating is closer than the focal point of the second lens. A minimum pulse width occurs near $-5.8 \mathrm{~cm}$ corresponding to a $1.62 \mathrm{ps} / \mathrm{nm}$ down-chirp from the laser.

optical phase was taken to be 0 , the phases of the modes on each side of this mode, progressively out from the center, would be $0.07,0.28$, and $0.62 \mathrm{rad}$, meaning that the standing waves for each of these modes would reach their peaks at earlier times corresponding to these optical phase shifts than would the center mode.

To verify these cross-correlation measurements, a set of standard autocorrelations was taken. Replacing the beam splitters in Fig. 1 with mirrors, the pulse width for a variety of positions of the telescoped compressor's second grating was measured. With all points plotted and fit with the expected function $\tau\left(1+a x^{2}\right)^{1 / 2}$, again, the minimum chirp position is shown to be inside the focal length of the second lens by about $5.8 \mathrm{~cm}$. The curve, confirming the measured down-chirp, is shown in Fig. 4.

The down-chirp depends on the injection current. As the relative strength of the absorber and gain sections is changed the amount of phase modulation from both the gain and absorber are expected to change, causing a different chirp. In fact, the down-chirp was found to increase at larger injection currents. The results of the chirp measurement for injection currents of $18 \mathrm{~mA}, 20 \mathrm{~mA}, 30 \mathrm{~mA}$, and $40 \mathrm{~mA}$ into the gain section are shown in Fig. 5. The colliding-pulse mode (CPM) effect, which has been observed in dye lasers and suggested [12] and studied [13] in semiconductor lasers, was not noticeable here. Two possible reasons for the absence of a transient grating are noted. The effectiveness of transient 


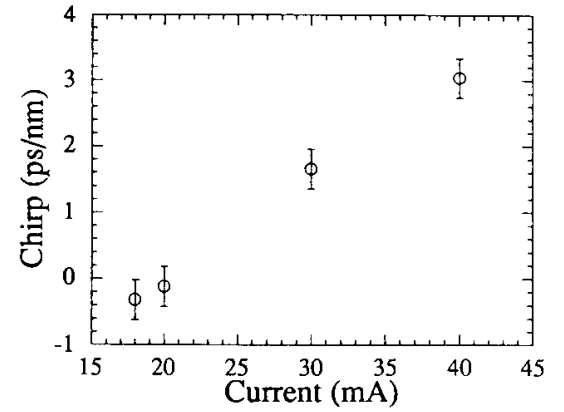

Fig. 5. Measured values of chirp as a function of gain-section current. An increasing down-chirp is seen as the gain current is increased.

grating buildup is expected to be proportional to both the peak intensity and the diffusion time constant. This product is rather small for peak cavity intensities around $5 \mathrm{MW} / \mathrm{cm}^{2}$ (about $1 / 100$ of that from external cavity lasers) and a relatively short diffusion time. The ambipolar diffusion constant $D^{*}=$ $15 \mathrm{~cm}^{2} / \mathrm{sec}$ for GaAs, results in a carrier diffusion time [14] of $t_{d} \approx L^{2} / 16 D^{*}=0.6 \mathrm{ps}$ for a grating to smear out to a full width of $L=\lambda / 2 n$, where $n=3.6$ is the material's index of refraction. This implies that the transient grating decays in a time shorter than the pulse width. Saturation effects, however, are a cumulative result from a number of pulses, since the repetition rate is faster than the recovery time of either section. Thus, for high-repetition rate lasers, the grating is expected to be blurred, even while the saturation effects are significant. Secondly, the $70 \%$ reflection provides incomplete standing wave interference for building the saturation grating. Nevertheless, without significant saturation grating effects, chirp-free pulses were obtained at the lower injection currents.

\section{CONCLUSION}

In conclusion, we have generated stable pulse trains of down-chirped and chirp-free pulses from high-repetition rate, passively mode-locked semiconductor lasers. Slightly upchirped and chirp-free pulses have been obtained at lower gain-section injection currents, while strongly down-chirped pulses have resulted from higher injection currents. The changing phase modulation effects are attributed to a change in the ratio of absorption to gain strengths. The latter pulses will undergo compression in fiber propagation at this wavelength.
A self-consistent analysis of the "supermode" structure of the high-repetition-rate lasers is to be published elsewhere [4].

\section{ACKNOWLEDGMENT}

The authors would like to acknowledge J. O'Brien and J. Kitching for helpful discussions about transient gratings in semiconductors, and T. R. Chen for providing reflection coatings.

\section{REFERENCES}

[1] J. F. Martins-Filho and C. N. Ironside, "Multiple colliding pulse modelocked operation of a semiconductor laser," Appl. Phys. Lett., vol. 65, pp. 1894-1896, Oct. 1994.

[2] S. Arahira, S. Oshiba, Y. Matsui, T. Kunii, and Y. Ogawa, "Terahertzrate optical pulse generation from a passively mode-locked semiconductor laser diode," Opt. Lett., vol. 19, pp. 834-836, June 1994.

[3] J. Paslaski and K. Y. Lau, "Parameter ranges for ultra high frequency mode locking of semiconductor lasers," Appl. Phys. Lett., vol. 59, pp. 7-9, July 1991.

[4] R. A. Salvatore, S. Sanders, T. Schrans, and A. Yariv, "Supermodes of high-repetition-rate passively mode-locked semiconductor lasers," IEEE J. Quantum Electron., submitted.

[5] K. Vahala and A. Yariv, "Semiclassical theory of noise in semiconductor lasers-Part I," IEEE J. Quantum Electron., vol. 19, pp. 1096-1101, June 1983.

[6] M. Y. Hong, Y. H. Chang, A. Dienes, J. P. Heritage, and P. J. Delfyett, "Subpicosecond pulse amplification in semiconductor laser amplifiers: Theory and experiment," IEEE J. Quantum Electron., vol. 30, pp. 1122-1131, Apr. 1994.

[7] G. P. Agrawal and N. A. Olsson, "Self-phase modulation and spectral broadening of optical pulses in semiconductor laser amplifiers," IEEE J. Quantum Electron., vol. 25, pp. 2297-2306, Nov. 1989.

[8] A. Azouz, N. Stelmakh, and J.-M. Lourtioz, "Passive mode locking of semiconductor lasers with tunable group velocity dispersion cavity," Electron. Lett., vol. 29, pp. 1437-1438, Aug. 1993.

[9] O. E. Martinez, R. L. Fork, and J. P. Gordon, "Theory of passively mode-locked lasers for the case of a nonlinear complex-propagation coefficient," J. Opt. Soc. Am. B, vol. 2, pp. 753-766, May 1985.

[10] J. L. A. Chilla and O. E. Martinez, "Analysis of a method of phase measurement of ultra short pulses in the frequency domain," IEEE $J$. Quantum Electron., vol. 27, pp. 1228-1235, May 1991.

[11] R. A. Salvatore, T. Schrans, and A. Yariv, "Pulse characteristics of passively mode-locked diode lasers," Opt. Lett., vol. 20, pp. 737-739, Apr. 1995.

[12] Y. K. Chen and M. C. Wu, "Monolithic colliding-pulse quantum-well lasers," IEEE J. Quantum Electron., vol. 28, pp. 2176-2185, Oct. 1992.

[13] D. J. Derickson, R. J. Helkey, A. Mar, J. R. Karin, J. G. Wasserbauer, and J. E. Bowers, "Short pulse generation usingm ultisegment modelocked semiconductor lasers," IEEE J. Quantum Electron., vol. 28, pp. 2186-2201, Oct. 1992.

[14] C. M. Wolfe, N. Holonyak, and G. E. Stillman, Physical Properties of Semiconductors. Englewood Cliffs, NJ: Prentice-Hall, 1989. 